\title{
REGRESSION METHODS FOR HESITANT FUZZY PREFERENCE RELATIONS
}

\author{
Bin ZHU, Zeshui XU \\ a School of Economics and Management, Southeast University, Nanjing, 211189 Jiangsu, China \\ ${ }^{\mathrm{b}}$ Business School, Sichuan University, Chengdu, 610064 Sichuan, China
}

Received 20 May 2012; accepted 18 November 2012

\begin{abstract}
In this paper, we develop two regression methods that transform hesitant fuzzy preference relations (HFPRs) into fuzzy preference relations (FPRs). On the basis of the complete consistency, reduced FPRs with the highest consistency levels can be derived from HFPRs. Compared with a straightforward method, this regression method is more efficient in the Matlab environment. Based on the weak consistency, another regression method is developed to transform HFPRs into reduced FPRs which satisfy the weak consistency. Two algorithms are proposed for the two regression methods, and some examples are provided to verify the practicality and superiority of the proposed methods.
\end{abstract}

Keywords: hesitant fuzzy preference relation (HFPR), fuzzy preference relation (FPR), complete consistency, weak consistency, consistency level.

Reference to this paper should be made as follows: Zhu, B.; Xu, Z. 2013. Regression methods for hesitant fuzzy preference relations, Technological and Economic Development of Economy 19(Supplement 1): S214-S227.

JEL Classification: C81; D7; D8.

\section{Introduction}

Fuzzy preference relations (FPRs) are widely used in decision making, where consistency of FPRs is a major goal and interesting research topic (Herrera-Viedma et al. 2004, 2007; Jiang, Fan 2008; Tanino 1984, 1988; Wu et al. 2012; Wei et al. 2012; Stankevičienè, Mencaitė 2012; Baležentis et al. 2012). Recently, hesitant fuzzy sets (HFSs), originally introduced by Torra (2010), become a hot topic (Zhu et al. 2012a, b, 2013; Xu, Xia 2011). HFSs can consider the

Corresponding author Bin Zhu

E-mail: binzhu@263.net 
degrees of membership by a set of possible values. The motivation to propose HFSs is that when defining the membership of an element, the difficulty of establishing the membership degree is not a margin of error (as in intuitionistic fuzzy sets; Atanassov 1986), or some possibility distributions on the possible values (as in type 2 fuzzy sets; Zadeh 1975), but a set of possible values (Torra 2010).

With respect to the preference relations of HFSs, Xia and Xu (2013) defined hesitant fuzzy preference relations (HFPRs) and developed an approach to apply HFPRs to decision making. However, as a basic issue of HFPRs, the studies on consistency of HFPRs is not easy because the numbers of possible values in different hesitant fuzzy elements (HFEs) are often different. Since FPRs have been proven to be an effective tool used in decision making problems (Chiclana et al. 2001; Orlovsky 1978; Tanino 1984), we consider some techniques to transform HFPRs into FPRs based on their close relationship. Two regression methods are developed for the transformations based on the complete consistency and the weak consistency respectively.

The rest of this paper is organized as follows. Section 1 reviews some basic knowledge. In Section 2, we develop the regression methods, and illustrate their advantages with some examples. The final section ends the paper with some conclusions.

\section{Preliminaries}

This section introduces some concepts related to hesitant fuzzy sets (HFSs), fuzzy preference relations (FPRs), and hesitant fuzzy preference relations (HFPRs).

\subsection{Hesitant fuzzy preference relations}

Torra (2010) originally developed HFSs which cover arguments with a set of possible values.

Definition 1 (Torra 2010). Let $X$ be a fixed set, a hesitant fuzzy set (HFS) on $X$ is in terms of a function that when applied to $X$ returns a subset of $[0,1]$.

To be easily understood, Xia and Xu (2011) expressed the HFS by a mathematical symbol:

$$
E=\{<x, h(x)>\mid x \in X\}
$$

where $h(x)$ is a set of some values in [0,1], denoting the possible membership degrees of the element $x \in X$ to the set $E$. For convenience, Xia and Xu (2011) called $h$ a hesitant fuzzy element (HFE).

For a HFE $h$, Xia and Xu (2011) developed some operations as follows:

1) $h^{\lambda}=\bigcup_{\gamma \in h}\left\{\gamma^{\lambda}\right\}, \lambda>0$;

2) $\lambda h=\bigcup_{\gamma \in h}\left\{1-(1-\gamma)^{\lambda}\right\}, \lambda>0$.

FPRs (Orlovsky 1978) are an effective tool in decision making. The definition is as follows.

Definition 2 (Orlovsky 1978). A fuzzy preference relation (FPR) $P$ on a set of objectives, $X$, is a fuzzy set on the product set $X \times X$, that is characterized by a membership function $\mu_{p}: X \times X \rightarrow[0,1]$. 
When the cardinality of $X$ is small, the fuzzy preference relation may be conveniently represented by a $n \times n$ matrix $P=\left(p_{i j}\right)_{n \times n}$, where $p_{i j}=\mu_{p}\left(x_{i}, x_{j}\right) . p_{i j}$ is interpreted as the preference degree of the objective $x_{i}$ over $x_{j}: p_{i j}=0.5$ indicates indifference between $x_{i}$ and $x_{j}$, which can be represented by $x_{i} \sim x_{j} ; p_{i j}=1$ indicates that $x_{i}$ is absolutely preferred $x_{j}$; $p_{i j}>0.5$ indicates that $x_{i}$ is preferred to $x_{j}$, which can be represented by $x_{i} \succ x_{j}$. Generally, $P$ is assumed to be additive reciprocal: $p_{i j}+p_{j i}=1, i, j=1,2, \ldots, n$.

On the basis of FPRs, Xia and Xu (2013) developed HFPRs which can be restated as follows.

Definition 3. Let $X=\left\{x_{1}, x_{2}, \ldots, x_{n}\right\}$ be a fixed set, then a hesitant fuzzy preference relation (HFPR) $H$ on $X$ is presented by a matrix $H=\left(h_{i j}\right)_{n \times n} \subset X \times X$, where $h_{i j}=\left\{\gamma_{i j}^{l} \mid l=1, \ldots, \# h_{i j}\right\}$ ( $\# h_{i j}$ is the number of values in $h_{i j}$ ) is a HFE indicating all the possible preference degree(s) of the objective $x_{i}$ over $x_{j}$. Moreover, $h_{i j}$ should satisfy the following conditions:

$$
\gamma_{i j}^{\sigma(l)}+\gamma_{j i}^{\sigma\left(\# h_{i j}-l+1\right)}=1, \gamma_{i i}=\{0.5\}, \# h_{i j}=\# h_{j i}, i, j=1,2, \ldots, n,
$$

where: $\gamma_{i j}^{\sigma(l)}$ is the $l$ th largest element in $h_{i j}$.

\subsection{Consistency measures}

The transitivity property is used to represent the idea that the preference degree obtained by directly comparing two objectives should be equal to or greater than the preference degree between those two objectives obtained using an indirect chain of objectives. This property is desirable to avoid contradictions reflected in preference relations. For the FPR $P=\left(p_{i j}\right)_{n \times n}$, Tanino (1984) introduced an additive fuzzy transitivity property, or called the complete consistency:

$$
p_{i j}+p_{j k}=p_{i k}+0.5
$$

Tanino (1988) also introduced an additive fuzzy weak transitivity, or called the weak consistency: $p_{i j} \geq 0.5, p_{j k} \geq 0.5 \rightarrow p_{i k} \geq 0.5, i, j, k=1, \ldots, n$. It means that if $x_{i}$ is preferred to $x_{j}$ and $x_{j}$ is preferred to $x_{k}$, then $x_{i}$ should be preferred to $x_{k}$. This property verifies the condition that a logical and consistent person does not want to express his/her opinions with inconsistency, which guarantee the minimum requirement for consistency.

\section{Regression methods for HFPRs}

In this section, we develop two regression methods for HFPRs, which depend on the complete consistency and the weak consistency respectively.

\subsection{A regression method for HFPRs based on the complete consistency}

Herrera-Viedma et al. (2007) developed a method with error analysis to measure the consistency levels of FPRs. Motivated by this method, and based on the complete consistency and error analysis, we develop a regression method to transform HFPRs into FPRs. 
Given a HFPR, represented by a matrix $H=\left(h_{i j}\right)_{n \times n} \subset X \times X$, where $X=\left\{x_{1}, x_{2}, \ldots, x_{n}\right\}$ is a fixed set of objectives. According to the definition of the complete consistency, the possible preference degrees over the paired objectives $(i, k)$ represented by a HFE $h_{i k}(i \neq k)$ can be estimated using an intermediate objective $x_{j}(j \neq i, k)$ :

$$
h_{i k}^{j}=h_{i j} \tilde{+} h_{j k} \simeq 0.5 \text {, }
$$

where: $h_{i k}^{j}$ can be called an estimated HFE, and the operations “ $\tilde{+}$ " and “ $\cong$ " are efined as follows.

Definition 4. Let $h, h_{1}$ and $h_{2}$ be three HFEs, and $a$ be a real number, then we define

$$
\begin{gathered}
h_{1} \tilde{+} h_{2}=\bigcup_{\gamma_{1} \in h_{1}, \gamma_{2} \in h_{2}}\left\{\gamma_{1}+\gamma_{2}\right\} ; \\
h \simeq a=\bigcup_{\gamma \in h}\{\gamma-a\} .
\end{gathered}
$$

In order to use Eq. (4) to estimate $h_{i k}^{j}$, the objectives $x_{i}(i=1,2, \ldots, n)$ should generally be classified into several sets defined as follows:

$$
\begin{gathered}
B=\{(i, k) \mid i, k \in\{1,2, \ldots, n\} \wedge(i \neq k)\} ; \\
O V^{B}=\{(i, k) \in B\} ; \\
K V^{B}=\left(O V^{B}\right)^{c} ; \\
M_{i k}^{j}=\left\{j \neq i, k \mid(i, j),(j, k) \in K V^{B}\right\},
\end{gathered}
$$

where: $B$ is a set of all paired objectives; $O V^{B}$ is a set of paired objectives $(i, k) ; K V^{B}$ is the complement set of $O V^{B}$ satisfying $K V^{B} \cup O V^{B}=B ; M_{i k}^{j}$ is the set of the intermediate objectives $x_{j}(j \neq i, k)$.

Based on the discussions above and according to Eq.(4), we can get all the estimated HFE $h_{i k}^{j}(j=1,2, \ldots n ; j \neq i, k)$. To select the optimal preference degree from $h_{i k}^{j}(j=1,2, \ldots n ; j \neq i, k)$, we calculate an average estimated preference degree defined as follows:

$$
h_{i k}^{A}=\frac{S_{s}\left(\bigcup_{j \in M_{i k}^{j}} h_{i k}^{j}\right)}{\sum_{j \in M_{i k}^{j}}\left(\# h_{i k}^{j}\right)},
$$

where: $S_{s}$ is a function that indicates the summation of all elements in a set; $\# h_{i k}^{j}$ indicates the numbers of possible preference degrees in $h_{i k}^{j}$.

Comparing the possible values in the HFE $h_{i k}$ and its average estimated preference degree $h_{i k}^{A}$, we define the error between them as follows. 
Definition 5. For the HFE $h_{i k}$ and its average estimated preference degree $h_{i k}^{A}$, the error between them is defined as:

$$
\varepsilon h_{i k}=\frac{2}{3}\left(\bigcup_{\varepsilon_{i k} \in\left(h_{i k} \simeq h_{i k}^{A}\right)}\left|\varepsilon_{i k}\right|\right),
$$

where: the coefficient $2 / 3$ is used to make sure each value of the error belongs to the unit interval $[0,1]$.

If there exists a preference degree $h_{i k}^{*}\left(h_{i k}^{*} \in h_{i k}\right)$ that corresponds to the minimum value of the error $\varepsilon h_{i k}$ satisfying:

$$
\frac{2}{3}\left(h_{i k}^{*}-h_{i k}^{A}\right)=\min \left(\varepsilon h_{i k}\right)
$$

then we should choose this preference degree as the optimal one. Following this principle and collecting $h_{i k}^{*}$ for all $i, k=1,2, \ldots, n ; i \neq k$, we can transform $H$ into a FPR $H^{*}=\left(h_{i k}^{*}\right)_{n \times n}$, which can be called a reduced FPR.

Further to measure the consistency level of $H^{*}$, we now give some definitions.

Definition 6. For the reduced FPR $H^{*}=\left(h_{i k}^{*}\right)_{n \times n}$, the consistency level of $h_{i k}^{*}$ is defined as:

$$
c l_{i k}=1-\min \left(\varepsilon h_{i k}\right) .
$$

With respect to one objective $x_{i}$, the consistency level is defined as follows.

Definition 7. For $H^{*}=\left(h_{i k}^{*}\right)_{n \times n}$, the consistency level of the objective $x_{i}$ is defined as:

$$
c l_{i}=\frac{\sum_{i \neq k, k=1}^{n}\left(c l_{i k}+c l_{k i}\right)}{2(n-1)} .
$$

So the consistency level of $H^{*}$ can be further defined with respect to all the objectives.

Definition 8. For $H^{*}=\left(h_{i k}^{*}\right)_{n \times n}$, its consistency level is defined as:

$$
c l_{H^{*}}=\frac{\sum_{i=1}^{n} c l_{i}}{n} \text {. }
$$

Clearly, the bigger the value of $c l_{H^{*}}\left(c l_{H^{*}} \in[0,1]\right)$, the higher the consistency level of $H^{*}$. Based on the analysis above, for a fixed set $X=\left\{x_{1}, x_{2}, \cdots, x_{n}\right\}$, and a constructed HFPR $H=\left(h_{i j}\right)_{n \times n}$, the algorithm that transforms $H$ into $H^{*}$ is shown in Algorithm $\mathrm{I}$.

\section{Algorithm I}

Step 1. Randomly locate a HFE $h_{i k}(i \neq k)$, then calculate $h_{i k}^{j}(j=1,2, \ldots n ; j \neq i, k)$ according to Eq. (4).

Step 2. Calculate the average estimated preference degree $h_{i k}^{A}$ by Eq. (11), and then obtain $h_{i k}^{*}$ by Eqs. (12) and (13).

Step 3. Repeat Steps 1 and 2 until all HFEs have been located, then turn to the next Step. 
Step 4. Collecting $h_{i k}^{*}$ for all $i, k=1,2, \ldots, n(i \neq k)$, we can get the reduced FPR $H^{*}=\left(h_{i k}^{*}\right)_{n \times n}$.

Step 5. Calculate the consistency level of $H^{*}$ according to Eqs. (14), (15) and (16).

Step 6. End.

Example 1. Assume a HFPR as follows:

$$
H_{1}=\left(\begin{array}{cccc}
\{0.5\} & \{0.4,0.5\} & \{0.6,0.7\} & \{0.6\} \\
\{0.5,0.6\} & \{0.5\} & \{0.8\} & \{0.4\} \\
\{0.3,0.4\} & \{0.2\} & \{0.5\} & \{0.1,0.2\} \\
\{0.4\} & \{0.6\} & \{0.8,0.9\} & \{0.5\}
\end{array}\right) .
$$

Step 1. Locate the HFE $h_{12}$, and according to Eq. (4), we have:

$$
h_{12}^{3}=h_{13} \tilde{+} h_{32} \simeq 0.5=\{0.3,0.4\}, h_{12}^{4}=h_{14} \tilde{+} h_{42} \simeq 0.5=\{0.7\} .
$$

Step 2. According to Eq. (11), we have:

$$
h_{12}^{A}=\frac{S_{s}\left(\sum_{j \in M_{i k}^{j}} h_{12}^{j}\right)}{\sum_{j \in M_{12}^{j}}\left(\# h_{12}^{j}\right)}=\frac{(0.3+0.4)+0.7}{2+1}=0.467 .
$$

By Eqs. (12) and (13), we can get:

$$
\varepsilon h_{12}=\frac{2}{3}\left(\bigcup_{\varepsilon_{12} \in\left(h_{12} \sim h_{12}^{A}\right)}\left|\varepsilon_{12}\right|\right)=\{0.044,0.022\}, \min \left(\varepsilon h_{12}\right)=0.022=\frac{2}{3}|0.5-0.467| \text {. }
$$

Thus, $h_{12}^{*}=0.5$.

Step 3. Repeat Steps 1 and 2, we have:

$$
\begin{gathered}
h_{13}^{*}=0.7, \min \left(\varepsilon h_{13}\right)=0.100 ; h_{14}^{*}=0.6, \min \left(\varepsilon h_{14}\right)=0.189 ; \\
h_{21}^{*}=0.5, \min \left(\varepsilon h_{21}\right)=0.022 ; h_{23}^{*}=0.8, \min \left(\varepsilon h_{23}\right)=0.056 ; \\
h_{24}^{*}=0.4, \min \left(\varepsilon h_{24}\right)=0.100 ; h_{31}^{*}=0.3, \min \left(\varepsilon h_{31}\right)=0.067 ; \\
h_{32}^{*}=0.2, \min \left(\varepsilon h_{32}\right)=0.056 ; h_{34}^{*}=0.2, \min \left(\varepsilon h_{34}\right)=0.089 ; \\
h_{41}^{*}=0.4, \min \left(\varepsilon h_{41}\right)=0.189 ; h_{42}^{*}=0.6, \min \left(\varepsilon h_{42}\right)=0.100 ; \\
h_{43}^{*}=0.8, \min \left(\varepsilon h_{43}\right)=0.089 .
\end{gathered}
$$

Step 4. Collecting $h_{i k}^{\star}$ for all $i, k=1,2, \ldots n(i \neq k)$, we can get the reduced FPR $H_{1}^{*}$ as:

$$
H_{1}^{*}=\left(\begin{array}{cccc}
0.5 & 0.5 & 0.7 & 0.6 \\
0.5 & 0.5 & 0.8 & 0.4 \\
0.3 & 0.2 & 0.5 & 0.2 \\
0.4 & 0.6 & 0.8 & 0.5
\end{array}\right) \text {. }
$$


Step 5. According to Eq. (14), we have:

$$
\begin{aligned}
& c l_{12}=0.978 ; c l_{21}=0.978 ; c l_{13}=0.900 ; c l_{31}=0.933 ; c l_{14}=0.811 ; c l_{41}=0.811 ; \\
& c l_{23}=0.944 ; c l_{32}=0.944 ; c l_{24}=0.900 ; c l_{42}=0.900 ; c l_{34}=0.911 ; c l_{43}=0.911 .
\end{aligned}
$$

According to Eq. (15), we can get:

$$
\begin{aligned}
& c l_{1}=\frac{\left(c l_{12}+c l_{21}\right)+\left(c l_{13}+c l_{31}\right)+\left(c l_{14}+c l_{41}\right)}{6}=0.902 ; \\
& c l_{2}=\frac{\left(c l_{21}+c l_{12}\right)+\left(c l_{23}+c l_{32}\right)+\left(c l_{24}+c l_{42}\right)}{6}=0.941 ; \\
& c l_{3}=\frac{\left(c l_{31}+c l_{13}\right)+\left(c l_{32}+c l_{23}\right)+\left(c l_{34}+c l_{43}\right)}{6}=0.924 ; \\
& c l_{4}=\frac{\left(c l_{41}+c l_{14}\right)+\left(c l_{42}+c l_{24}\right)+\left(c l_{43}+c l_{34}\right)}{6}=0.874 .
\end{aligned}
$$

Furthermore, by Eq. (16), the consistency level of $H_{1}^{*}$ is:

$$
c l_{H_{1}^{*}}=\frac{c l_{1}+c l_{2}+c l_{3}+c l_{4}}{4}=0.91 \text {. }
$$

Step 6. End.

For the HFPR $H=\left(h_{i j}\right)_{n \times n}$, since each preference degree in $h_{i j}$ is a possible value, $H$ can be directly separated into all possible FPRs. Then based on some existing consistency measure methods, the FPR with the highest consistency level can be found out. In order to compare this straightforward method with our method, we give the following example.

Example 2. Based on the same HFPR $H_{1}$, we can generate eight possible FPRs from $H_{1}$ denoted as follows:

$$
\begin{aligned}
& P_{1}^{H_{1}}=\left(\begin{array}{cccc}
0.5 & 0.5 & 0.6 & 0.6 \\
0.5 & 0.5 & 0.8 & 0.4 \\
0.4 & 0.2 & 0.5 & 0.1 \\
0.4 & 0.6 & 0.9 & 0.5
\end{array}\right) ; P_{2}^{H_{1}}=\left(\begin{array}{cccc}
0.5 & 0.5 & 0.7 & 0.6 \\
0.5 & 0.5 & 0.8 & 0.4 \\
0.3 & 0.2 & 0.5 & 0.1 \\
0.4 & 0.6 & 0.9 & 0.5
\end{array}\right) ; P_{3}^{H_{1}}=\left(\begin{array}{cccc}
0.5 & 0.5 & 0.6 & 0.6 \\
0.5 & 0.5 & 0.8 & 0.4 \\
0.4 & 0.2 & 0.5 & 0.2 \\
0.4 & 0.6 & 0.8 & 0.5
\end{array}\right) \text {; } \\
& P_{4}^{H_{1}}=\left(\begin{array}{cccc}
0.5 & 0.5 & 0.7 & 0.6 \\
0.5 & 0.5 & 0.8 & 0.4 \\
0.3 & 0.2 & 0.5 & 0.2 \\
0.4 & 0.6 & 0.8 & 0.5
\end{array}\right) ; P_{5}^{H_{1}}=\left(\begin{array}{cccc}
0.5 & 0.4 & 0.6 & 0.6 \\
0.6 & 0.5 & 0.8 & 0.4 \\
0.4 & 0.2 & 0.5 & 0.1 \\
0.4 & 0.6 & 0.9 & 0.5
\end{array}\right) ; P_{6}^{H_{1}}=\left(\begin{array}{cccc}
0.5 & 0.4 & 0.7 & 0.6 \\
0.6 & 0.5 & 0.8 & 0.4 \\
0.3 & 0.2 & 0.5 & 0.1 \\
0.4 & 0.6 & 0.9 & 0.5
\end{array}\right) ; \\
& P_{7}^{H_{1}}=\left(\begin{array}{cccc}
0.5 & 0.4 & 0.6 & 0.6 \\
0.6 & 0.5 & 0.8 & 0.4 \\
0.4 & 0.2 & 0.5 & 0.2 \\
0.4 & 0.6 & 0.8 & 0.5
\end{array}\right) ; P_{8}^{H_{1}}=\left(\begin{array}{cccc}
0.5 & 0.4 & 0.7 & 0.6 \\
0.6 & 0.5 & 0.8 & 0.4 \\
0.3 & 0.2 & 0.5 & 0.2 \\
0.4 & 0.6 & 0.8 & 0.5
\end{array}\right) \text {. }
\end{aligned}
$$


According to the consistency measure of FPRs introduced by Herrera-Viedma et al. (2007), we can get the consistency levels of $P_{i}^{H_{1}} \quad(i=1,2, \ldots, 8)$ denoted by:

$$
\begin{gathered}
c_{P_{1}^{H_{1}}}=89.63 \% ; c_{P_{2}^{H_{1}}}=91.76 \% ; c_{P_{3}^{H_{1}}}=90.56 \% ; c_{P_{4}^{H_{1}}}=92.69 \% ; \\
c_{P_{5}^{H_{1}}}=88.52 \% ; c l_{P_{6} H_{1}}=90.65 \% ; c_{P_{7}^{H_{1}}}=89.44 \% ; c_{P_{8}^{H_{1}}}=91.57 \% .
\end{gathered}
$$

Since:

$$
c_{P_{4}^{H_{1}}}>c l_{P_{2}^{H_{1}}}>c l_{P_{8}^{H_{1}}}>c l_{P_{6}^{H_{1}}}>c l_{P_{3}^{H_{1}}}>c l_{P_{1}^{H_{1}}}>c l_{P_{7}^{H_{1}}}>c l_{P_{5}^{H_{1}}},
$$

$P_{4}^{H_{1}}$ is with the highest consistency level.

Obviously, $P_{4}^{H_{1}}=H_{1}^{*}$. So the results are the same by the regression method and the straightforward method. Considering the efficiency, the number of operations of our regression method and the straightforward method are $2 n(n-1)+n+1$ and $m(n(n-1)+n+1)(m$ is the number of all possible FPRs separated from a HFPR), respectively. Since $m \geq 2$ (at least two FPRs can be separated from a HFPR), we have $m(n(n-1)+n+1)>2 n(n-1)+n+1$. So our method is simpler. Moreover, the bigger the value $m$, the simpler the regression method.

\subsection{A regression method for HFPRs based on the weak consistency}

For the decision making problems in practical applications, the complete consistency is sometimes not necessary due to the complicated environment and the cognitive diversity of humans. But, the weak consistency is essential because a contradictory HFPR doesn't make sense. On the basis of the weak consistency, we now develop another regression method to get reduced FPRs satisfying the weak consistency. In what follows, we begin with some necessary definitions and discussions.

Definition 9. Assume a HFPR, $H=\left(h_{i j}\right)_{n \times n}=\bigcup_{\gamma_{i j} \in h_{i j}}\left\{\gamma_{i j}\right\}_{n \times n}$, its hesitant preference degree (HPD) is defined as:

$$
m_{i j}=\bigcup_{s_{i j} \in m_{i j}^{H}}\left\{s_{i j}\right\}
$$

where: $s_{i j}$ is called a hesitant preference element (HPE), satisfying:

$$
s_{i j}=\left\{\begin{array}{l}
1, \text { if } 0.5<\gamma_{i j} \leq 1 \\
0, \text { if } 0 \leq \gamma_{i j}<0.5
\end{array} .\right.
$$

Then $M=\left(m_{i j}\right)_{n \times n}$ is called a hesitant preference relation (HPR).

According to graph theory (Bondy, Murty 1976), the relationship included in the HPR can be described by a directed graph which can be called a hesitant fuzzy preference graph. In such a graph, each node stands for an objective, and each directed edge stands for a preference relation. If $m_{i j}=1$, then there is a directed edge from a node $i$ to a node $j$, which represents that the objective $i$ is superior to the objective $j$. 
Example 3. Assume a fixed set $X=\left\{x_{1}, x_{2}, x_{3}, x_{4}\right\}$, and two constructed HFPRs as

$$
H_{2}=\left(\begin{array}{cccc}
\{0.5\} & \{0.6\} & \{0.6,0.7\} & \{0.6\} \\
\{0.4\} & \{0.5\} & \{0.8\} & \{0.4\} \\
\{0.3,0.4\} & \{0.2\} & \{0.5\} & \{0.2\} \\
\{0.4\} & \{0.6\} & \{0.8\} & \{0.5\}
\end{array}\right) ; H_{3}=\left(\begin{array}{cccc}
\{0.5\} & \{0.4,0.6\} & \{0.6\} & \{0.4,0.6\} \\
\{0.4,0.6\} & \{0.5\} & \{0.8\} & \{0.4\} \\
\{0.4\} & \{0.2\} & \{0.5\} & \{0.2,0.3\} \\
\{0.4,0.6\} & \{0.6\} & \{0.7,0.8\} & \{0.5\}
\end{array}\right) .
$$

According to Definition 9, we get:

$$
M_{H_{2}}=\left(\begin{array}{cccc}
\{0\} & \{1\} & \{1\} & \{1\} \\
\{0\} & \{0\} & \{1\} & \{0\} \\
\{0\} & \{0\} & \{0\} & \{0\} \\
\{0\} & \{1\} & \{1\} & \{0\}
\end{array}\right) ; M_{H_{3}}=\left(\begin{array}{cccc}
\{0\} & \{1,0\} & \{1\} & \{1,0\} \\
\{0,1\} & \{0\} & \{1\} & \{0\} \\
\{0\} & \{0\} & \{0\} & \{0\} \\
\{0,1\} & \{1\} & \{1\} & \{0\}
\end{array}\right) .
$$

The hesitant fuzzy preference graphs of $M_{H_{2}}$ and $M_{H_{3}}$ are shown in Figures 1 and 2 respectively.

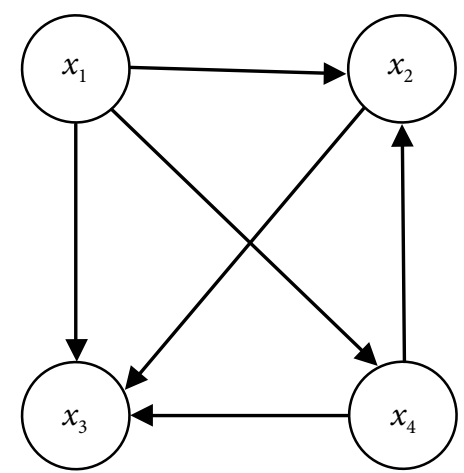

Fig. 1. Hesitant fuzzy preference graph of $M_{\mathrm{H}_{2}}$

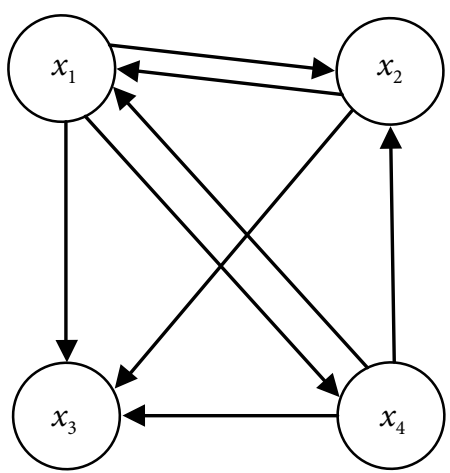

Fig. 2. Hesitant fuzzy preference graph of $M_{H_{3}}$

With respect to a HFPR, if there is no circular triad in a hesitant fuzzy preference graph, it means that a circular relation of objectives does not exist. So the HFPR satisfies the weak consistency, such as in Fig. 1. However, in Fig. 2, we can see that the objectives $x_{1}$ and $x_{4}$ are connected by two opposite directed edges. In such a case, we can get a circular triad of objectives as $x_{1} \rightarrow x_{4} \rightarrow x_{2} \rightarrow x_{1}$ in Fig. 2 . Thus the corresponding HFPR, $H_{3}$, does not satisfy the weak consistency. Therefore, the circular triad can be used to test the weak consistency of HFPRs, which is defined as follows. then:

Definition 10. Let $M=\left(m_{i j}\right)_{n \times n}$ betheHPRofaHFPR $H=\left(h_{i j}\right)_{n \times n}$, where $m_{i j}=\bigcup_{s_{i j} \in m_{i j}}\left\{s_{i j}\right\}$,

$$
C_{i j k}=\bigcup_{c_{i j k} \in C_{i j k}}\left\{c_{i j k}\right\}=\bigcup_{s_{i j} \in m_{i j}, s_{j k} \in m_{j k}, s_{k i} \in m_{k i}}\left\{s_{i j}+s_{j k}+s_{k i}\right\} \quad(i, j, k \in\{1,2, \ldots, n\} ; i \neq j \neq k),
$$

is called a circular triad power, and $c_{i j k}$ is called a circular triad power element. 
Theorem 1. For a HFPR, $H=\left(h_{i j}\right)_{n \times n}$, we can get its HPR $M=\left(m_{i j}\right)_{n \times n}$, and the circular triad power $C_{i j k}=\bigcup_{c_{i j k} \in C_{i j k}}\left\{c_{i j k}\right\}$. If and only if there exists $c_{i j k}=3$, then $H$ does not satisfy the weak consistency.

Proof. If $c_{i j k}=3$ exists, then there exists one circular triad indicating a relation of objectives as $x_{i} \succ x_{j} \succ x_{k} \succ x_{i}$. According to the definition of the weak consistency, $H$ does not satisfy the weak consistency; if $H$ does not satisfy the weak consistency, then there exists a circular triad of objectives as $x_{i} \rightarrow x_{j} \rightarrow x_{k} \rightarrow x_{i}$. According to Definition 10, there exists $c_{i j k}=3$, which complete the proof.

Jiang and Fan (2008) gave a definition of a reachability matrix used to test the weak consistency of FPRs. Motivated by this idea, and based on Theorem 1, we now develop a hesitant reachability matrix (HRM) to identify the weak consistency of HFPRs.

Definition 11. Let $M=\left(m_{i j}\right)_{n \times n}$ be the HPR of a HFPR $H=\left(h_{i j}\right)_{n \times n}$, then we call $M^{(k)}=\left(m_{i j}^{(k)}\right)_{n \times n}$ the $k$ th power of $M$, where the $(i, j)$ entry, denoted by $m_{i j}^{(k)}$, is the number of different directed edges of the length $k$ from the node $i$ to the node $j$.

Furthermore, we define the HRM as follows.

Definition 12. Let $M=\left(m_{i j}\right)_{n \times n}$ be the HPR of a HFPR $H=\left(h_{i j}\right)_{n \times n}, M^{(3)}=\left(m_{i j}^{(3)}\right)_{n \times n}$ be the third power of $M$, then we call the matrix $R=\left(r_{i j}\right)_{n \times n}$ the hesitant reachability matrix (HRM), where $R=M^{(3)}$.

Theorem 2. For a HFPR, $H=\left(h_{i j}\right)_{n \times n}$, if all diagonal elements are zero in its hesitant reachability matrix $R=\left(r_{i j}\right)_{n \times n}$, then $H$ satisfies the weak consistency.

Proof. For the HFPR, $H=\left(h_{i j}\right)_{n \times n}$, if all the diagonal elements are zero in its hesitant reachability matrix $R=\left(r_{i j}\right)_{n \times n}$, i.e. $M^{(3)}=\left(m_{i i}^{(3)}\right)_{n \times n}=0$, then according to Theorem 1 , we know that there is no circular triad in $H$. So $H$ satisfies the weak consistency, which completes the proof.

According to Definition 12, and based on the two HFPRs $H_{2}$ and $H_{3}$ in Example 3, we can get two HRMs as follows:

$$
R_{H_{2}}=\left(\begin{array}{cccc}
0 & 0 & 1 & 0 \\
0 & 0 & 0 & 0 \\
0 & 0 & 0 & 0 \\
0 & 0 & 0 & 0
\end{array}\right) ; R_{H_{3}}=\left(\begin{array}{cccc}
1 & 0 & 1 & 0 \\
0 & 1 & 1 & 0 \\
0 & 0 & 0 & 0 \\
0 & 0 & 2 & 1
\end{array}\right) \text {. }
$$

According to Theorem 2, $\mathrm{H}_{2}$ satisfies the weak consistency, but $\mathrm{H}_{3}$ does not.

Based on the discussions above, we now give a step by step procedure to obtain reduced FPRs satisfying the weak consistency shown in Algorithm $\Pi$.

\section{Algorithm ח}

Given a HFPR, $H^{p}=\left(h_{i j}^{p}\right)_{n \times n}\left(p=0 ; H^{p}\right.$ is the $p$ th power of $H$ indicating the number of being modified).

Step 1. According to Definitions 9 and 12, we can get its HPR $M^{p}=\left(m_{i j}\right)_{n \times n}$ and HRM $R^{p}=\left(r_{i j}\right)_{n \times n}$, respectively. 
Step 2. According to Theorem 2, if $H^{p}$ satisfies the weak consistency, turn to Step 5; otherwise, turn to Step 3.

Step 3. For the HPR $M^{p}=\left(m_{i j}\right)_{n \times n}$, where $m_{i j}=\bigcup_{s_{i j} \in m_{i j}}\left\{s_{i j}\right\}$, locate a pair of HPDs $\left(m_{i j}, m_{j i}\right)$, satisfying $m_{i j}=\{0,1\}, m_{j i}=\{1,0\}$.

According to Eq. (19), find out a $c_{i j k}=3$, remove the pair of HPEs $\left(s_{i j}, s_{j i}\right)$ satisfying $s_{i j}+s_{j i}=1$, and remove their corresponding preference degrees in the pair of HFEs $\left(h_{i j}, h_{j i}\right)$ in $H$.

Step 4. Let $p=p+1$, construct a modified HFPR as $H^{p+1}$, turn to Step 1 .

Step 5. Divide $H^{p}$ into all possible reduced FPRs.

Step 6. End.

Example 4. Continued with $H_{3}$ in Example 3, let $H_{3}$ be $H_{3}^{p}(p=0)$. Since $H_{3}^{p}$ does not satisfy the weak consistency, we turn to Step 3.

Step 3. Locate $\left(m_{14}, m_{41}\right)$, satisfying $m_{14}=\{0,1\}$ and $m_{41}=\{1,0\}$. According to Eq. (19), we can find $c_{142}=3$. Remove the pair of HPEs $s_{14}=\{0\}$ and $s_{41}=\{1\}$, and remove their corresponding preference degrees $\gamma_{14}=0.4, \gamma_{41}=0.6$ in the pair of HFEs $\left(h_{14}, h_{41}\right)$.

Step 4. Let $p=p+1$, construct a modified HFPR $H_{3}^{1}$ as follows:

$$
H_{3}^{1}=\left(\begin{array}{cccc}
\{0.5\} & \{0.4,0.6\} & \{0.6\} & \{0.4\} \\
\{0.4,0.6\} & \{0.5\} & \{0.8\} & \{0.4\} \\
\{0.4\} & \{0.2\} & \{0.5\} & \{0.2,0.3\} \\
\{0.6\} & \{0.6\} & \{0.7,0.8\} & \{0.5\}
\end{array}\right) .
$$

Then turn to Step 1.

Step 1. According to Definitions 9 and 12, and the modified HHPR $H_{3}^{1}$, we can get:

$$
M_{H_{3}^{1}}=\left(\begin{array}{cccc}
\{0\} & \{1,0\} & \{1\} & \{0\} \\
\{0,1\} & \{0\} & \{1\} & \{0\} \\
\{0\} & \{0\} & \{0\} & \{0\} \\
\{1\} & \{1\} & \{1\} & \{0\}
\end{array}\right) ; R_{H_{3}^{1}}=\left(\begin{array}{cccc}
0 & 0 & 0 & 0 \\
0 & 0 & 0 & 0 \\
0 & 0 & 0 & 0 \\
0 & 0 & 1 & 0
\end{array}\right) .
$$

Step 2. According to Theorem $2, H_{3}^{1}$ satisfies the weak consistency, turn to Step 5.

Step 5. Divide $H_{3}^{1}$ into the following possible reduced FPRs satisfying the weak consistency:

$$
\begin{aligned}
& P_{1}^{H_{3}^{1}}=\left(\begin{array}{llll}
0.5 & 0.4 & 0.6 & 0.4 \\
0.6 & 0.5 & 0.8 & 0.4 \\
0.4 & 0.2 & 0.5 & 0.2 \\
0.6 & 0.6 & 0.8 & 0.5
\end{array}\right) ; P_{2}^{H_{3}^{1}}=\left(\begin{array}{cccc}
0.5 & 0.4 & 0.6 & 0.4 \\
0.6 & 0.5 & 0.8 & 0.4 \\
0.4 & 0.2 & 0.5 & 0.3 \\
0.6 & 0.6 & 0.7 & 0.5
\end{array}\right) ; \\
& P_{3}^{H_{3}^{1}}=\left(\begin{array}{llll}
0.5 & 0.6 & 0.6 & 0.4 \\
0.4 & 0.5 & 0.8 & 0.4 \\
0.4 & 0.2 & 0.5 & 0.2 \\
0.6 & 0.6 & 0.8 & 0.5
\end{array}\right) ; P_{4}^{H_{3}^{1}}=\left(\begin{array}{cccc}
0.5 & 0.6 & 0.6 & 0.4 \\
0.4 & 0.5 & 0.8 & 0.4 \\
0.4 & 0.2 & 0.5 & 0.3 \\
0.6 & 0.6 & 0.7 & 0.5
\end{array}\right)
\end{aligned}
$$


Step 6. End.

In practical applications, Algorithms I and $\Pi$ can be combined to obtain reduced FPRs from HFPRs, where the obtained reduced FPR can not only satisfy the weak consistency but also have the highest confidence level.

For example, we replace Step 5 in Example 4 by Algorithm I. Then we can obtain a reduced FPR, denoted by $H_{2}^{\star}$, with the highest consistency level $95.56 \%$ :

\section{Conclusions}

$$
H_{2}^{*}=\left(\begin{array}{llll}
0.5 & 0.4 & 0.6 & 0.4 \\
0.6 & 0.5 & 0.8 & 0.4 \\
0.4 & 0.2 & 0.5 & 0.2 \\
0.6 & 0.6 & 0.8 & 0.5
\end{array}\right)
$$

For a hesitant fuzzy preference relation (HFPR), it is not easy to deal with its consistency due to different numbers of possible values in hesitant fuzzy elements (HFEs). In this paper, we have developed two regression methods to transform HFPRs into reduced fuzzy preference relations (FPRs). Based on the complete consistency, we use error analysis to select the optimal preference degree for each paired objectives in HFPRs to produce reduced FPRs. The step by step procedure of this regression method is shown in Algorithm I. On the basis of the weak consistency, we have defined a hesitant preference relation (HPR) and a circular triad power to find circular triads of objectives in HFPRs. Then we have given Theorems 1 and 2 to identify the weak consistency of HFPRs. With these definitions and methods, we have given Algorithm $\Pi$ to transform HFPRs into FPRs that satisfy the weak consistency.

\section{Acknowledgements}

The authors would like to thank the anonymous referees for their insightful and constructive comments and suggestions that have led to an improved version of this paper. The work was supported by the National Natural Science Foundation of China (No.61273209), the Fundamental Research Funds for the Central Universities (No. CXZZ12_0132), the excellent $\mathrm{PhD}$ thesis Foundation of Southeast University, and the Best New PhD Foundation of China.

\section{References}

Atanassov, K. 1986. Intuitionistic fuzzy sets, Fuzzy Sets and Systems 20(1): 87-96. http://dx.doi.org/10.1016/S0165-0114(86)80034-3

Baležentis, A.; Baležentis, T.; Misiunas, A. 2012. An integrated assessment of Lithuanian economic sectors based on financial ratios and fuzzy MCDM methods, Technological and Economic Development of Economy 18(1): 34-53. http://dx.doi.org/10.3846/20294913.2012.656151

Bondy, J. A.; Murty, U. S. R. 1976. Graph theory with applications. The Macmillan Press Ltd., Great Britain. $270 \mathrm{p}$. 
Chiclana, F.; Herrera, F.; Herrera-Viedma, E. 2001. Integrating multiplicative preference relations in a multipurpose decision-making model based on fuzzy preference relations, Fuzzy Sets and Systems 122(2): 277-291. http://dx.doi.org/10.1016/S0165-0114(00)00004-X

Herrera-Viedma, E.; Alonso, S.; Chiclana, F.; Herrera, F. 2007. A consensus model for group decision making with incomplete fuzzy preference relations, IEEE Transactions on Fuzzy Systems 15(5): 863-877. http://dx.doi.org/10.1109/TFUZZ.2006.889952

Herrera-Viedma, E.; Herrera, F.; Chiclana, F.; Luque, M. 2004. Some issues on consistency of fuzzy preference relations, European Journal of Operational Research 154(1): 98-109. http://dx.doi.org/10.1016/S0377-2217(02)00725-7

Jiang, Y. P.; Fan, Z. P. 2008. Judgment matrix: theory and methods. Beijing: Science Press.

Orlovsky, S. A. 1978. Decision-making with a fuzzy preference relation, Fuzzy Sets and Systems 1(3): 155-167. http://dx.doi.org/10.1016/0165-0114(78)90001-5

Stankevičienè, J.; Mencaite, E. 2012. The evaluation of bank performance using a multicriteria decision making model: a case study on Lithuanian commercial banks, Technological and Economic Development of Economy 18(1): 189-205. http://dx.doi.org/10.3846/20294913.2012.668373

Tanino, T. 1984. Fuzzy preference orderings in group decision making, Fuzzy Sets and Systems 12(2): 117-131. http://dx.doi.org/10.1016/0165-0114(84)90032-0

Tanino, T. 1988. Fuzzy preference relations in group decision making, in Kacprzyk, J.; Roubens, M. (Eds.). Non-Conventional Preference Relations in Decision Making 1988. Springer-Verlag, Berlin, 54-71. http://dx.doi.org/10.1007/978-3-642-51711-2_4

Torra, V. 2010. Hesitant fuzzy sets, International Journal of Intelligent Systems 25(6): 529-539.

Wei, G. W.; Zhao, X. F.; Wang, H. J. 2012. An approach to multiple attribute group decision making with interval intuitionistic trapezoidal fuzzy information, Technological and Economic Development of Economy 18(2): 317-330. http://dx.doi.org/10.3846/20294913.2012.676995

Wu, W. S.; Kou, G.; Peng, Y.; Ergu, D. J. 2012. Improved AHP-group decision making for investment strategy selection, Technological and Economic Development of Economy 18(2): 299-316. http://dx.doi.org/10.3846/20294913.2012.680520

Xia, M. M.; Xu, Z. S. 2011. Hesitant fuzzy information aggregation in decision making, International Journal of Approximate Reasoning 52(3): 395-407. http://dx.doi.org/10.1016/j.ijar.2010.09.002

Xia, M. M.; Xu, Z. S. 2013. Managing hesitant information in GDM problems under fuzzy and multiplicative preference relations, International Journal of Uncertainty, Fuzziness and Knowledge-Based Systems 21(6): 865. http://dx.doi.org/10.1142/S0218488513500402

Xu, Z. S.; Xia, M. M. 2011. Distance and similarity measures for hesitant fuzzy sets, Information Sciences 181(11): 2128-2138. http://dx.doi.org/10.1016/j.ins.2011.01.028

Zadeh, L. A. 1975. The concept of a linguistic variable and its application to approximate reasoning, Part I, Information Sciences 8(3): 199-249. http://dx.doi.org/10.1016/0020-0255(75)90036-5

Zhu, B.; Xu, Z. S.; Xia, M. M. 2012a. Hesitant fuzzy geometric Bonferroni means, Information Sciences 205(1): 72-85. http://dx.doi.org/10.1016/j.ins.2012.01.048

Zhu, B.; Xu, Z. S.; Xia, M. M. 2012b. Dual hesitant fuzzy sets, Journal of Applied Mathematics 2012: 1-13. http://dx.doi.org/10.1155/2012/879629

Zhu, B.; Xu, Z. S.; Xu. J. P. 2013. Deriving a ranking from hesitant fuzzy preference relations under group decision making, IEEE Transactions on Systems, Man, and Cybernetics, Part B: Cybernetics PP(99): 1 . http://dx.doi.org/10.1109/TCYB.2013.2283021 
Bin ZHU received the Bachelor's degree from Southeast University, China, in 2008. He is currently working toward the PhD degree with the School of Economics and Management, Southeast University. His research results have been published in Fuzzy Sets and Systems, Information Sciences, IEEE Transactions on Fuzzy Systems, IEEE Transactions on Systems, Man, and Cybernetics, Part B: Cybernetics, Journal of the Operational Research Society, Knowledge-Based Systems among others. His research interests include decision analysis, decision support, information fusion, and computing with words.

Zeshui XU received the PhD degree in Management Science and Engineering from Southeast University, Nanjing, China, in 2003. From April 2003 to May 2005, he was a Postdoctoral Researcher with School of Economics and Management, Southeast University. From October 2005 to December 2007, he was a Postdoctoral Researcher with School of Economics and Management, Tsinghua University, Beijing, China. He is a Distinguished Young Scholar of the National Natural Science Foundation of China, and a Distinguished Professor of the Chang Jiang Scholars Program of the Ministry of Education of China. He is with the Business School, Sichuan University, Chengdu, China. He has authored seven monographs and contributed more than 350 journal articles to professional journals. He is currently the Associate Editor of Fuzzy Optimization and Decision Making, Journal of Intelligence Systems, and also a member of Editorial Boards of seventeen professional journals. His current research interests include information fusion, group decision making, computing with words, and aggregation operators. 